\title{
Net charge fluctuations in AA collisions in a simple string-inspired model
}

\author{
Arsenii Titov* \\ Department of High Energy and Elementary Particle Physics, Faculty of Physics, \\ Saint Petersburg State University, Saint Petersburg, Russia \\ E-mail: arsenii.titovegmail.com

\section{Vladimir Vechernin} \\ Department of High Energy and Elementary Particle Physics, Faculty of Physics, \\ Saint Petersburg State University, Saint Petersburg, Russia \\ E-mail: vecherninepobox.spbu.ru
}

\begin{abstract}
We consider a simple model of net charge fluctuations in high-energy nucleus-nucleus collisions inspired by the string picture of hadronic interactions. It is found that in this model the so-called $D$ measure of the net charge fluctuations used by Jeon and Koch depends only on the parameters characterizing the charge fluctuations inside a string and does not depend on the event-by-event fluctuations of the number of strings.
\end{abstract}

XXI International Baldin Seminar on High Energy Physics Problems September 10-15, 2012

JINR, Dubna, Russia

\footnotetext{
* Speaker.
} 


\section{Introduction}

Net charge event-by-event fluctuations have been proposed as one of the indicators of the formation of quark-gluon plasma (QGP) in high-energy nucleus-nucleus collisions [ [W, ㅁ] . Various measures of these fluctuations have been introduced in the literature [U, [1, 目, 田]. Advantages and

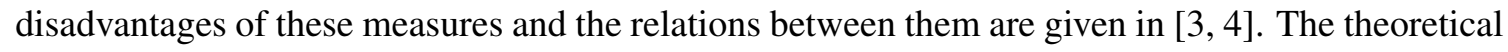
predictions of the value of the fluctuations [U, 口, [] are not directly consistent with the experimental results from RHIC [圆. The attempts have been made to take into account the influence of the different effects on the value of the net charge fluctuations (see for example [ [ $\mathbb{Z}, \mathbb{8}]$ ). At present the preliminary results extracted from the ALICE experimental data have rather ambiguous interpretation in the framework of existing models [Q [0] $]$. In this connection, we try to describe the experimental data in the framework of an alternative string-inspired model.

The soft part of multiparticle production at high energy is successfully explained by the string models [ए], [2], [3]]. In these models a hadron production is described in the framework of a two-stage scenario. At the first stage a certain number of quark-gluon strings (colour field tubes) stretched between the projectile and target partons are formed. At the second stage quark-antiquark pairs are created from a vacuum by the colour field transforming these strings into the chains of observed hadrons. It is important that those processes are dominant, in which in a chain the produced hadrons are ordered in rapidity with a constant density. It means that in string models one has an approximate conservation of the charge locally in rapidity. The deviation of the charge created in rapidity interval $\Delta y$ from zero is due to the processes, when on the boarders of this interval only one charged particle (of the created pair with close rapidities) belongs to the interval $\Delta y$.

In string models the positive charge (and the baryon number) of initial nuclear protons is associated with their valence quarks and is concentrated at projectile and target rapidities. A transfer of this charge to the mid-rapidity region $\Delta y$ is suppressed in the Gribov-Regge picture of the soft hadron interactions as corresponding to the exchange of reggeons with non-vacuum quantum numbers, so the suppression increases with energy. However, the process of the initial nuclear charge (baryon number) transfer to the mid-rapidity region $\Delta y$ can be enhanced at high energies due to the hard processes (the "stoppage" of valence quarks) if their contribution increases with energy. This transfer is the second cause of the deviation of the charge from zero in the mid-rapidity region.

Based on this picture of hadronic interactions we formulate a simple model of the net charge fluctuations.

\section{Formulation of the model}

The observable $v$ is determined as the second moment of the difference between the relative multiplicities $N_{+} /\left\langle N_{+}\right\rangle$and $N_{-} /\left\langle N_{-}\right\rangle$in a given phase space volume as follows:

$$
v \equiv\left\langle\left(\frac{N_{+}}{\left\langle N_{+}\right\rangle}-\frac{N_{-}}{\left\langle N_{-}\right\rangle}\right)^{2}\right\rangle .
$$

If the particle distributions are independent from each other, i.e.,

$$
\left\langle N_{+} N_{-}\right\rangle=\left\langle N_{+}\right\rangle\left\langle N_{-}\right\rangle
$$


and Poissonian, i.e.,

$$
\begin{aligned}
& D\left(N_{+}\right) \equiv\left\langle N_{+}^{2}\right\rangle-\left\langle N_{+}\right\rangle^{2}=\left\langle N_{+}\right\rangle, \\
& D\left(N_{-}\right) \equiv\left\langle N_{-}^{2}\right\rangle-\left\langle N_{-}\right\rangle^{2}=\left\langle N_{-}\right\rangle,
\end{aligned}
$$

the quantity $v$ is equal to so-called $v_{\text {stat }}$ :

$$
v_{\mathrm{stat}}=\frac{1}{\left\langle N_{+}\right\rangle}+\frac{1}{\left\langle N_{-}\right\rangle}
$$

where the subscript "stat" stands for "statistical". The "dynamical" fluctuations is the difference between the above two quantities:

$$
v_{\mathrm{dyn}}=v-v_{\mathrm{stat}} \text {. }
$$

This measure of the net charge fluctuations has been studied by the STAR Collaboration at RHIC [目] and the ALICE Collaboration at the LHC [ए]]

The quantity $v_{\text {dyn }}$ can be written as follows:

$$
v_{\text {dyn }}=\frac{D\left(N_{+}\right)-\left\langle N_{+}\right\rangle}{\left\langle N_{+}\right\rangle^{2}}+\frac{D\left(N_{-}\right)-\left\langle N_{-}\right\rangle}{\left\langle N_{-}\right\rangle^{2}}-2 \frac{\left\langle N_{+} N_{-}\right\rangle-\left\langle N_{+}\right\rangle\left\langle N_{-}\right\rangle}{\left\langle N_{+}\right\rangle\left\langle N_{-}\right\rangle} .
$$

In order to calculate the mean values $\left\langle N_{+}\right\rangle,\left\langle N_{-}\right\rangle$, the variances $D\left(N_{+}\right), D\left(N_{-}\right)$, and the covariance $\left\langle N_{+} N_{-}\right\rangle-\left\langle N_{+}\right\rangle\left\langle N_{-}\right\rangle$we adopt the formalism used for calculating of long-range (forward-

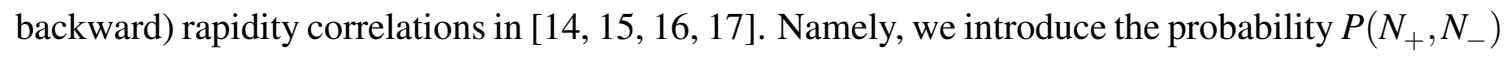
to have $N_{+}$positive and $N_{-}$negative particles in some rapidity interval $\Delta y$ in a given event due to a fragmentation of $N$ strings, independent between themselves:

$$
P\left(N_{+}, N_{-}\right)=\sum_{N} w(N) \sum_{n_{1}^{+}, \ldots, n_{N}^{+}} \sum_{n_{1}^{-}, \ldots, n_{N}^{-}} \delta_{N_{+}} n_{1}^{+}+\ldots+n_{N}^{+} \delta_{N_{-} n_{1}^{-}+\ldots+n_{N}^{-}} \prod_{i=1}^{N} p\left(n_{i}^{+}, n_{i}^{-}\right) .
$$

Here $w(N)$ is the probability of the formation of $N$ strings in a given event, $p\left(n_{i}^{+}, n_{i}^{-}\right)$is the probability to have $n_{i}^{+}$and $n_{i}^{-}$particles from the decay of the $i$-th string in the rapidity interval $\Delta y$ in a given event. It is important to note that we consider that the probability $p\left(n_{i}^{+}, n_{i}^{-}\right)$does not factorize into the product of two probabilities, i.e., the corresponding particle distributions are not independent. Then the following relations take place:

$$
\begin{array}{lll}
p_{+}\left(n_{i}^{+}\right)=\sum_{n_{i}^{-}} p\left(n_{i}^{+}, n_{i}^{-}\right), & \sum_{n_{i}^{+}} p_{+}\left(n_{i}^{+}\right)=1, \\
p_{-}\left(n_{i}^{-}\right)=\sum_{n_{i}^{+}} p\left(n_{i}^{+}, n_{i}^{-}\right), & & \sum_{n_{i}^{-}} p_{-}\left(n_{i}^{-}\right)=1 .
\end{array}
$$

For simplicity, we assume that all strings, formed in AA collisions at given conditions, are identical. Then at any $i$ we have

$$
\begin{gathered}
\sum_{n_{i}^{+}} n_{i}^{+} p_{+}\left(n_{i}^{+}\right)=\bar{n}_{+}, \quad \sum_{n_{i}^{+}} n_{i}^{+2} p_{+}\left(n_{i}^{+}\right)=\overline{n_{+}^{2}} \\
\sum_{n_{i}^{-}} n_{i}^{-} p_{-}\left(n_{i}^{-}\right)=\bar{n}_{-}, \quad \sum_{n_{i}^{-}} n_{i}^{-2} p_{-}\left(n_{i}^{-}\right)=\overline{n_{-}^{2}} \\
\sum_{n_{i}^{+}, n_{i}^{-}} n_{i}^{+} n_{i}^{-} p\left(n_{i}^{+}, n_{i}^{-}\right)=\overline{n_{+} n_{-}}
\end{gathered}
$$


We define the correlator for one string as follows:

$$
k \equiv \frac{\overline{n_{+} n_{-}}-\bar{n}_{+} \bar{n}_{-}}{\sqrt{d_{+} d_{-}}}
$$

where

$$
d_{+} \equiv \overline{n_{+}^{2}}-\bar{n}_{+}^{2}, \quad d_{-} \equiv \overline{n_{-}^{2}}-\bar{n}_{-}^{2}
$$

are the variances of the numbers of positive and negative particles from one string. As it follows from definition (2.8)

$$
-1 \leq k \leq 1 .
$$

The stronger the correlation between positive and negative particles produced due to the fragmentation of a string the more the absolute value of the correlator. If the particle distributions are independent from each other, then the correlator is equal to zero.

The fluctuations in the number of strings are characterized by $w(N)$ :

$$
\sum_{N} w(N)=1, \quad \sum_{N} N w(N)=\langle N\rangle, \quad \sum_{N} N^{2} w(N)=\left\langle N^{2}\right\rangle, \quad D(N)=\left\langle N^{2}\right\rangle-\langle N\rangle^{2} .
$$

Here $\langle N\rangle$ is the mean number of strings, and $D(N)$ is the variance of the number of strings. Note that these fluctuations are strongly non-Poissonian [18]].

Now we can calculate the mean values, the variances, and the covariance of $N_{+}$and $N_{-}$. Using (2.5), ([2.6), and (2.7), we have

$$
\begin{aligned}
\left\langle N_{+}\right\rangle & =\sum_{N_{+}, N_{-}} N_{+} P\left(N_{+}, N_{-}\right) \\
& =\sum_{N_{+}, N_{-}} N_{+} \sum_{N} w(N) \sum_{n_{1}^{+}, \ldots, n_{N}^{+} n_{1}^{-}, \ldots, n_{N}^{-}} \delta_{N_{+}} n_{1}^{+}+\ldots+n_{N}^{+} \delta_{N_{-}} n_{1}^{-}+\ldots+n_{N}^{-} \prod_{i=1}^{N} p\left(n_{i}^{+}, n_{i}^{-}\right) \\
& =\sum_{N} w(N) \sum_{n_{1}^{+}, \ldots, n_{N}^{+}} \sum_{n_{1}^{-}, \ldots, n_{N}^{-}}\left(n_{1}^{+}+\ldots+n_{N}^{+}\right) \prod_{i=1}^{N} p\left(n_{i}^{+}, n_{i}^{-}\right) \\
& =\sum_{N} w(N) \sum_{n_{1}^{+}, \ldots, n_{N}^{+}}\left(n_{1}^{+}+\ldots+n_{N}^{+}\right) \prod_{i=1}^{N} p_{+}\left(n_{i}^{+}\right)=\sum_{N} w(N) N \bar{n}_{+}=\langle N\rangle \bar{n}_{+} .
\end{aligned}
$$

Similarly, we obtain

$$
\left\langle N_{-}\right\rangle=\langle N\rangle \bar{n}_{-},
$$

and

$$
\begin{aligned}
& D\left(N_{+}\right)=\langle N\rangle d_{+}+D(N) \bar{n}_{+}^{2}, \\
& D\left(N_{-}\right)=\langle N\rangle d_{-}+D(N) \bar{n}_{-}^{2},
\end{aligned}
$$

and, using also (2.8),

$$
\left\langle N_{+} N_{-}\right\rangle-\left\langle N_{+}\right\rangle\left\langle N_{-}\right\rangle=\langle N\rangle k \sqrt{d_{+} d_{-}}+D(N) \bar{n}_{+} \bar{n}_{-} .
$$

For the derivation of (2.11)-(‥13) see the Appendix $⿴$. 
At high energy one can assume that in the mid-rapidity region the mean number $\bar{n}_{+}$of positive particles from one string is equal to the mean number $\bar{n}_{-}$of negative ones. It is reasonable to suppose that the corresponding variances are equal to each other too. Let us denote

$$
\bar{n} \equiv \bar{n}_{+}=\bar{n}_{-} \quad \text { and } \quad d \equiv d_{+}=d_{-} .
$$

Then we can rewrite $([2)-.([] 3$.$) as follows:$

$$
\begin{gathered}
\left\langle N_{+}\right\rangle=\left\langle N_{-}\right\rangle=\langle N\rangle \bar{n}, \\
D\left(N_{+}\right)=D\left(N_{-}\right)=\langle N\rangle d+D(N) \bar{n}^{2}, \\
\left\langle N_{+} N_{-}\right\rangle-\left\langle N_{+}\right\rangle\left\langle N_{-}\right\rangle=\langle N\rangle k d+D(N) \bar{n}^{2} .
\end{gathered}
$$

One can note that at such assumptions

$$
\langle Q\rangle=0,
$$

where $Q=N_{+}-N_{-}$is the net charge, and

$$
\left\langle N_{\mathrm{ch}}\right\rangle=2\langle N\rangle \bar{n},
$$

where $N_{\mathrm{ch}}=N_{+}+N_{-}$is the charged particle multiplicity. Substituting (2.14)-(12.16) in (2.4), we obtain

$$
v_{\mathrm{dyn}}=\frac{2}{\langle N\rangle \bar{n}}(\omega(1-k)-1)
$$

where

$$
\omega \equiv \frac{d}{\bar{n}}
$$

is the scaled variance. Thus, the measure $v_{\mathrm{dyn}}$ is expressed through the parameters characterizing the individual string and the mean number of strings and does not depend on the variance of the number of strings, i.e., on the event-by-event fluctuations of this number.

The $D$ measure used by Jeon and Koch [U] and $v_{\text {dyn }}$ are related to each other by [एप]:

$$
\left\langle N_{\mathrm{ch}}\right\rangle v_{\mathrm{dyn}} \approx D-4
$$

By $(\mathbb{2 . 1 8})$ and (2.19) we have

$$
\left\langle N_{\mathrm{ch}}\right\rangle v_{\mathrm{dyn}}=4 \omega(1-k)-4 .
$$

This quantity does not depend on the parameters of the event-by-event distribution of the number of strings. In the following section we focus on rapidity dependence of the net charge fluctuations.

\section{Rapidity dependence}

In this section we address to dependence of $v_{\mathrm{dyn}}$ on the width of the central rapidity interval $\Delta y$. For this reason, we should find out the dependencies on $\Delta y$ of all quantities the expression (2.19) for $v_{\text {dyn }}$ consists of. We suppose that all strings formed in AA collision give a contribution to the mid-rapidity region $\Delta y$, and, therefore, the mean number $\langle N\rangle$ of strings does not depend 
on $\Delta y$. The following ingredient is the mean multiplicity $\bar{n}$ of positive (negative) particles from a string. At small values of $\Delta y$ one can consider that

$$
\bar{n}=\bar{n}_{0} \Delta y
$$

where $\bar{n}_{0}$ is the constant positive (negative) particle multiplicity density (the mean number of positive (negative) particles per unit rapidity).

The rapidity dependence of the scaled variance $\omega$ can be related with (3. a distribution of the number of positive (negative) particles in rapidity is uniform. In the case of the uniform distribution the doubly scaled variance $(\omega-1) / \bar{n}$ does not depend on $\Delta y$ (see formula (15) in [20] and formula (10) in [저, see also the Appendix [B]). So we can write:

$$
\frac{\omega-1}{\bar{n}}=C,
$$

where $C$ is independent on $\Delta y$. Then by (3., and (3.2) we have

$$
\omega=C \bar{n}_{0} \Delta y+1
$$

The more sophisticated question is a functional shape of the correlator $k$. Motivated by the string fragmentation picture [D2, [23, [24], we use the following approximation:

$$
k=1-\exp \left(-\frac{\Delta y}{\lambda_{0}}\right)
$$

where $\lambda_{0} \sim 1$ is the correlation length in rapidity. By (B.4) we consider that the correlation between the number of positive particles and the number of negative ones, belonging to the rapidity interval $\Delta y$, grows with increasing width of this interval, and $k \rightarrow 0$, when $\Delta y \rightarrow 0$, and $k \rightarrow 1$, when $\Delta y \rightarrow \infty$. The correlation length $\lambda_{0}$ designates the growth rate of the correlator. The stronger the correlation the less the value of $\lambda_{0}$ for a given $\Delta y$.

Now we substitute (B.]), (B.3), (B.4) in ([.19), (2.211) and obtain

$$
\begin{gathered}
\nu_{\mathrm{dyn}}(\Delta y)=\frac{2}{\langle N\rangle \bar{n}_{0} \Delta y}\left(\left(C \bar{n}_{0} \Delta y+1\right) \exp \left(-\frac{\Delta y}{\lambda_{0}}\right)-1\right), \\
\left\langle N_{\mathrm{ch}}\right\rangle v_{\mathrm{dyn}}(\Delta y)=4\left(C \bar{n}_{0} \Delta y+1\right) \exp \left(-\frac{\Delta y}{\lambda_{0}}\right)-4 .
\end{gathered}
$$

\section{Poisson approximation}

In this section we assume that the positive (negative) particle production from the decay of one string obeys the Poisson distribution. For the Poisson distribution the mean value $\bar{n}$ is equal to the variance $d$, and the scaled variance $\omega=1$. So in the Poisson approximation by (2.19) and (2.2T) we have

$$
\begin{gathered}
v_{\text {dyn }}=-\frac{2 k}{\langle N\rangle \bar{n}}, \\
\left\langle N_{\mathrm{ch}}\right\rangle v_{\text {dyn }}=-4 k .
\end{gathered}
$$


In this approximation $C=0($ see (B.2) $)$ in (B.5) and (B.6). Then we have

$$
\begin{gathered}
v_{\mathrm{dyn}}(\Delta y)=\frac{2}{\langle N\rangle \bar{n}_{0} \Delta y}\left(\exp \left(-\frac{\Delta y}{\lambda_{0}}\right)-1\right), \\
\left\langle N_{\mathrm{ch}}\right\rangle v_{\mathrm{dyn}}(\Delta y)=4 \exp \left(-\frac{\Delta y}{\lambda_{0}}\right)-4 .
\end{gathered}
$$

In [6] values of $v_{\mathrm{dyn}}(\Delta \eta) /\left|v_{\mathrm{dyn}}(2)\right|$ measured for different ranges of pseudorapidity $\eta$ are plotted. Here $v_{\text {dyn }}(2)$ is the magnitude of $v_{\text {dyn }}(\Delta \eta)$ for the pseudorapidity range $|\eta|<1(\Delta \eta=$ 2 ). In our formulae we can approximately replace the rapidity interval $\Delta y$ by the pseudorapidity interval $\Delta \eta$. Then, using (4.3]), for the normalized quantity, experimentally studied by the STAR Collaboration, we have

$$
\frac{v_{\mathrm{dyn}}(\Delta \eta)}{\left|v_{\mathrm{dyn}}(2)\right|}=\frac{2}{\Delta \eta} \cdot \frac{\exp \left(-\Delta \eta / \lambda_{0}\right)-1}{\left|\exp \left(-2 / \lambda_{0}\right)-1\right|}
$$

For this quantity we have only one parameter, the correlation length $\lambda_{0}$, which, nevertheless, enables to describe the experimental data obtained in [6].

In Fig. $\mathbb{W}$ the data for AuAu collisions at $\sqrt{s_{N N}}=62.4$ and $200 \mathrm{GeV}(0-5 \%$ centrality), $\mathrm{CuCu}$ collisions at $\sqrt{s_{N N}}=62.4$ and $200 \mathrm{GeV}(0-10 \%$ centrality), $p p$ collisions at $\sqrt{s}=200 \mathrm{GeV}$ obtained in [6] and the lines calculated with (4.5) are presented.

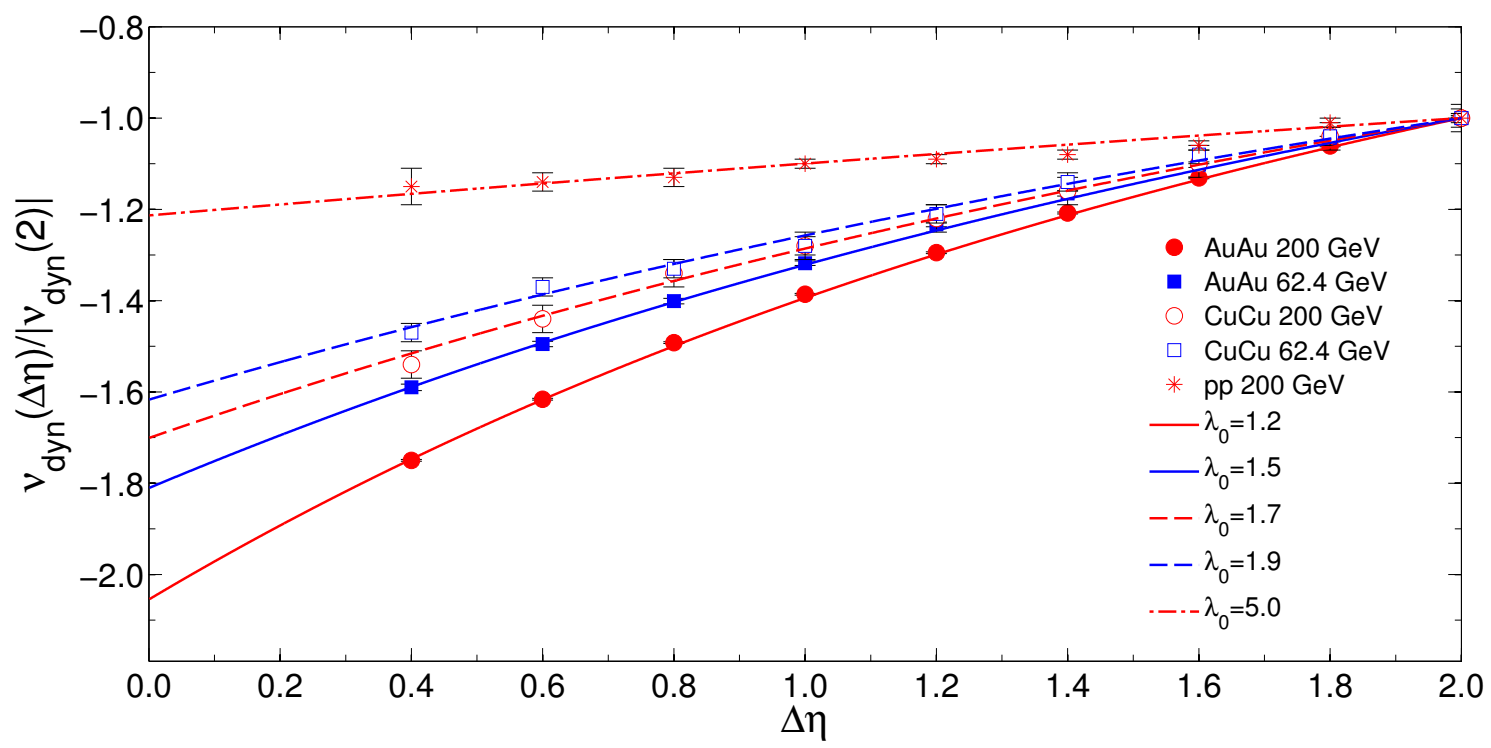

Figure 1: Dynamical fluctuations $v_{\mathrm{dyn}}$, normalized to their value for $|\eta|<1(\Delta \eta=2)$, as function of $\Delta \eta$. Data for AuAu collisions at $\sqrt{s_{N N}}=62.4,200 \mathrm{GeV}\left(0-5 \%\right.$ centrality), CuCu collisions at $\sqrt{s_{N N}}=$ $62.4,200 \mathrm{GeV}(0-10 \%$ centrality), $p p$ collisions at $\sqrt{s}=200 \mathrm{GeV}$ and lines calculated in the Poisson approximation of our model.

We can see that the correlation length $\lambda_{0}$ decreases with increasing energy and atomic number of colliding nuclei. In the framework of our model this can be interpreted as the formation of more intensive colour strings in AA collisions with the growth of both the energy and atomic number. 


\section{Summary and conclusions}

We formulated a simple model of the net charge fluctuations inspired by the string picture of hadronic interactions. The measure of the dynamical net charge fluctuations $v_{\text {dyn }}$ was expressed in terms of the parameters characterizing the individual string and their event-by-event distribution. It was shown, in particular, that the so-called $D$ measure of the net charge fluctuations used by Jeon and Koch [ $[$ depends only on the parameters characterizing the charge fluctuations inside a string and does not depend on the event-by-event fluctuations of the number of strings.

With some additional assumptions we found the dependence of these measures on the width of the mid-rapidity interval. Presuming that the positive (negative) particle production from one string obeys the Poisson distribution, we described the STAR data [目] with one parameter, the correlation length in rapidity. We found out that this parameter decreases with increasing energy and atomic number of colliding nuclei. In the framework of our model this fact means the formation of more intensive colour strings in nucleus-nucleus collisions with the growth of both the energy and atomic number.

\section{Acknowledgments}

We thank Grigory Feofilov and Urs Wiedemann for useful discussions. A.T. is supported by the Dynasty Foundation fellowship. V.V. is supported by the RFBR grant 12-02-00356-a.

\section{Appendices}

\section{A. Calculation of the variances and the covariance of the numbers of positive and negative particles}

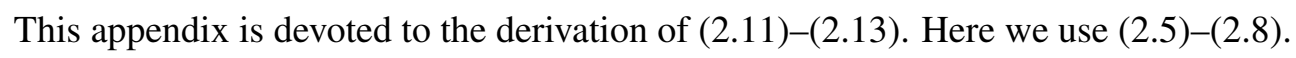

To calculate the variances

$$
D\left(N_{+}\right) \equiv\left\langle N_{+}^{2}\right\rangle-\left\langle N_{+}\right\rangle^{2}
$$

and

$$
D\left(N_{-}\right) \equiv\left\langle N_{-}^{2}\right\rangle-\left\langle N_{-}\right\rangle^{2}
$$

one should calculate $\left\langle N_{+}^{2}\right\rangle$ and $\left\langle N_{-}^{2}\right\rangle$.

$$
\begin{aligned}
\left\langle N_{+}^{2}\right\rangle & =\sum_{N_{+}, N_{-}} N_{+}^{2} P\left(N_{+}, N_{-}\right) \\
& =\sum_{N_{+}, N_{-}} N_{+}^{2} \sum_{N} w(N) \sum_{n_{1}^{+}, \ldots, n_{N}^{+}} \sum_{n_{1}^{-}, \ldots, n_{N}^{-}} \delta_{N_{+}} n_{1}^{+}+\ldots+n_{N}^{+} \delta_{N_{-}} n_{1}^{-}+\ldots+n_{N}^{-} \prod_{i=1}^{N} p\left(n_{i}^{+}, n_{i}^{-}\right) \\
& =\sum_{N} w(N) \sum_{n_{1}^{+}, \ldots, n_{N}^{+}} \sum_{n_{1}^{-}, \ldots, n_{N}^{-}}\left(n_{1}^{+}+\ldots+n_{N}^{+}\right)^{2} \prod_{i=1}^{N} p\left(n_{i}^{+}, n_{i}^{-}\right) \\
& =\sum_{N} w(N) \sum_{n_{1}^{+}, \ldots, n_{N}^{+}}\left\{\sum_{i=1}^{N} n_{i}^{+2}+\sum_{i \neq j=1}^{N} n_{i}^{+} n_{j}^{+}\right\} \prod_{i=1}^{N} p_{+}\left(n_{i}^{+}\right)
\end{aligned}
$$




$$
\begin{aligned}
& =\sum_{N} w(N)\left\{N \overline{n_{+}^{2}}+N(N-1) \bar{n}_{+}^{2}\right\}=\langle N\rangle \overline{n_{+}^{2}}+\left(\left\langle N^{2}\right\rangle-\langle N\rangle\right) \bar{n}_{+}^{2} \\
& =\langle N\rangle\left(\overline{n_{+}^{2}}-\bar{n}_{+}^{2}\right)+\left\langle N^{2}\right\rangle \bar{n}_{+}^{2}=\langle N\rangle d_{+}+\left\langle N^{2}\right\rangle \bar{n}_{+}^{2}
\end{aligned}
$$

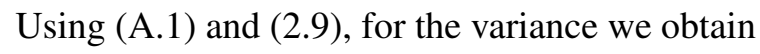

$$
D\left(N_{+}\right)=\langle N\rangle d_{+}+\left\langle N^{2}\right\rangle \bar{n}_{+}^{2}-\langle N\rangle^{2} \bar{n}_{+}^{2}=\langle N\rangle d_{+}+D(N) \bar{n}_{+}^{2} .
$$

Similarly,

$$
\left\langle N_{-}^{2}\right\rangle=\langle N\rangle d_{-}+\left\langle N^{2}\right\rangle \bar{n}_{-}^{2}
$$

and

$$
D\left(N_{-}\right)=\langle N\rangle d_{-}+D(N) \bar{n}_{-}^{2} .
$$

For the calculation of the covariance $\left\langle N_{+} N_{-}\right\rangle-\left\langle N_{+}\right\rangle\left\langle N_{-}\right\rangle$we need to know $\left\langle N_{+} N_{-}\right\rangle$.

$$
\begin{aligned}
& \left\langle N_{+} N_{-}\right\rangle=\sum_{N_{+}, N_{-}} N_{+} N_{-} P\left(N_{+}, N_{-}\right) \\
& =\sum_{N_{+}, N_{-}} N_{+} N_{-} \sum_{N} w(N) \sum_{n_{1}^{+}, \ldots, n_{N}^{+}} \sum_{n_{1}^{-}, \ldots, n_{N}^{-}} \delta_{N_{+}} n_{1}^{+}+\ldots+n_{N}^{+} \delta_{N_{-}} n_{1}^{-}+\ldots+n_{N}^{-} \prod_{i=1}^{N} p\left(n_{i}^{+}, n_{i}^{-}\right) \\
& =\sum_{N} w(N) \sum_{n_{1}^{+}, \ldots, n_{N}^{+}} \sum_{n_{1}^{-}, \ldots, n_{N}^{-}}\left(n_{1}^{+}+\ldots+n_{N}^{+}\right)\left(n_{1}^{-}+\ldots+n_{N}^{-}\right) \prod_{i=1}^{N} p\left(n_{i}^{+}, n_{i}^{-}\right) \\
& =\sum_{N} w(N) \sum_{n_{1}^{+}, \ldots, n_{N}^{+}} \sum_{n_{1}^{-}, \ldots, n_{N}^{-}}\left\{\sum_{i=1}^{N} n_{i}^{+} n_{i}^{-}+\sum_{i \neq j=1}^{N} n_{i}^{+} n_{j}^{-}\right\} \prod_{i=1}^{N} p\left(n_{i}^{+}, n_{i}^{-}\right) \\
& =\sum_{N} w(N)\left\{N \overline{n_{+} n_{-}}+N(N-1) \bar{n}_{+} \bar{n}_{-}\right\}=\langle N\rangle \overline{n_{+} n_{-}}+\left(\left\langle N^{2}\right\rangle-\langle N\rangle\right) \bar{n}_{+} \bar{n}_{-} \\
& =\langle N\rangle\left(\overline{n_{+} n_{-}}-\bar{n}_{+} \bar{n}_{-}\right)+\left\langle N^{2}\right\rangle \bar{n}_{+} \bar{n}_{-}=\langle N\rangle k \sqrt{d_{+} d_{-}}+\left\langle N^{2}\right\rangle \bar{n}_{+} \bar{n}_{-} .
\end{aligned}
$$

Using ( $(\overline{A .5})$ ), (‥9), and (2.10), for the covariance we obtain

$$
\begin{aligned}
\left\langle N_{+} N_{-}\right\rangle-\left\langle N_{+}\right\rangle\left\langle N_{-}\right\rangle & =\langle N\rangle k \sqrt{d_{+} d_{-}}+\left\langle N^{2}\right\rangle \bar{n}_{+} \bar{n}_{-}-\langle N\rangle^{2} \bar{n}_{+} \bar{n}_{-} \\
& =\langle N\rangle k \sqrt{d_{+} d_{-}}+D(N) \bar{n}_{+} \bar{n}_{-} .
\end{aligned}
$$

\section{B. Independence of the doubly scaled variance on the width of the rapidity interval}

In this appendix we mean that $n$ is $n_{i}^{+}$or $n_{i}^{-}$. Let us separate some subinterval $\Delta y^{\prime}$ belonging to the rapidity interval $\Delta y\left(\Delta y^{\prime} \subset \Delta y\right)$. Denote by $P(n)$ the probability of the production of $n$ particles within the acceptance $\Delta y$ and by $P^{\prime}\left(n^{\prime}\right)$ the probability of the production of $n^{\prime}$ particles within the acceptance $\Delta y^{\prime}$ in a given event. Let $p$ is the probability that the momentum of the particle produced in the rapidity interval $\Delta y$ also belongs to the subinterval $\Delta y^{\prime}$. In the case of independent particle production we have

$$
p=\frac{\left\langle n^{\prime}\right\rangle}{\langle n\rangle} .
$$


Note that in the case of the uniform distribution within $\Delta y$ we also have

$$
p=\frac{\left\langle n^{\prime}\right\rangle}{\langle n\rangle}=\frac{\Delta y^{\prime}}{\Delta y} .
$$

It is clear that we have the connection between $P(n)$ and $P^{\prime}\left(n^{\prime}\right)$ :

$$
P^{\prime}\left(n^{\prime}\right)=\sum_{n} P(n) P_{n}\left(n^{\prime}\right)
$$

where $P_{n}\left(n^{\prime}\right)$ is the probability that $n^{\prime}$ particles occur in the interval $\Delta y^{\prime}$, when $n$ particles are produced within the interval $\Delta y$. For the independent particle production it is given by the binomial distribution:

$$
P_{n}\left(n^{\prime}\right)=C_{n}^{n^{\prime}} p^{n^{\prime}}(1-p)^{n-n^{\prime}}
$$

(see the paragraph before formula (15) in [[20]).

The formulae $(\mathbb{B} .3)$ and $(\mathbb{B} .4)$ directly lead to the following relations:

$$
\begin{aligned}
\left\langle n^{\prime}\right\rangle & =\sum_{n^{\prime}} n^{\prime} P^{\prime}\left(n^{\prime}\right)=\sum_{n^{\prime}} \sum_{n} n^{\prime} P(n) P_{n}\left(n^{\prime}\right)=\sum_{n} P(n) p n=p\langle n\rangle, \\
\left\langle n^{\prime 2}\right\rangle & =\sum_{n^{\prime}} n^{\prime 2} P^{\prime}\left(n^{\prime}\right)=\sum_{n^{\prime}} \sum_{n} n^{\prime 2} P(n) P_{n}\left(n^{\prime}\right) \\
& =\sum_{n} P(n)\left(p^{2} n^{2}+p(1-p) n\right)=p^{2}\left\langle n^{2}\right\rangle+p(1-p)\langle n\rangle .
\end{aligned}
$$

We have used that for binomial distribution

$$
\overline{n^{\prime}}=p n, \quad D\left(n^{\prime}\right) \equiv \overline{n^{\prime 2}}-\overline{n^{\prime}}=p(1-p) n .
$$

The formulae $(\mathbb{B} .5)$ and $(\mathbb{B} .6)$ after simple algebra lead to

$$
\frac{\frac{D\left(n^{\prime}\right)}{\left\langle n^{\prime}\right\rangle}-1}{\left\langle n^{\prime}\right\rangle}=\frac{\omega\left(n^{\prime}\right)-1}{\left\langle n^{\prime}\right\rangle}=\frac{\omega(n)-1}{\langle n\rangle}=\frac{\frac{D(n)}{\langle n\rangle}-1}{\langle n\rangle}=\text { const },
$$

which does not depend on the width of the rapidity interval.

\section{References}

[1] S. Jeon and V. Koch, Charged Particle Ratio Fluctuation as a Signal for Quark-Gluon Plasma, Phys. Rev. Lett. 85 (2000) 2076 [hep-ph / 0003168$].$

[2] M. Asakawa, U. Heinz, and B. Müller, Fluctuation Probes of Quark Deconfinement, Phys. Rev. Lett. 85 (2000) 2072 [hep-ph / 0003169$].$

[3] C. Pruneau, S. Gavin, and S. Voloshin, Methods for the study of particle production fluctuations, Phys. Rev. C 66 (2002) 044904 [nucl-ex/ 0204011 ].

[4] S. Mrówczyński, Measuring charge fluctuations in high-energy nuclear collisions, Phys. Rev. C 66 (2002) 024904 [nucl-th/0112007].

[5] E.V. Shuryak and M.A. Stephanov, Long-range charge fluctuations and search for a quark-gluon plasma signal, Phys. Rev. C 63 (2001) 064903 [hep-ph / 0010100 ]. 
[6] B.I. Abelev et al. (STAR Collaboration), Beam-energy and system-size dependence of dynamical net charge fluctuations, Phys. Rev. C 79 (2009) 024906 [0807.3269 [nucl-ex]].

[7] M.A. Aziz and S. Gavin, Causal diffusion and the survival of charge fluctuations in nuclear collisions, Phys. Rev. C 70 (2004) 034905 [nucl-th/ 0404058 ].

[8] L. Shi and S. Jeon, Charge transfer fluctuations as a signal for quark-gluon plasma, Phys. Rev. C 72 (2005) 034904 [hep-ph/0503085].

[9] B. Abelev et al. (ALICE Collaboration), Net-charge fluctuations in Pb-Pb collisions at $\sqrt{s_{N N}}=2.76 \mathrm{TeV}, 1207.6068 \quad[$ nucl-ex $]$.

[10] S. Jena for the ALICE Collaboration, Charge fluctuations in Pb-Pb collisions at $\sqrt{s_{N N}}=2.76 \mathrm{TeV}$ measured by ALICE experiment, in proceedings of the Seventh Workshop on Particle Correlations and Femtoscopy, POS (WPCF2011)046[1203.0542 [nucl-ex]].

[11] A.B. Kaidalov, The quark-gluon structure of the pomeron and the rise of inclusive spectra at high energies, Phys. Lett. B 116 (1982) 459.

[12] A.B. Kaidalov, K.A. Ter-Martirosyan, Pomeron as quark-gluon strings and multiple hadron production at SPS-Collider energies, Phys. Lett. B 117 (1982) 247.

[13] A. Capella, U.P. Sukhatme, C.-I. Tan, J. Tran Thanh Van, Dual parton model, Phys. Rep. 236 (1994) 225.

[14] M.A. Braun, C. Pajares, V.V. Vechernin, On the forward-backward correlations in a two-stage scenario, Phys. Lett. B 493 (2000) 54 [hep-ph/ 0007241 ].

[15] M.A. Braun, R.S. Kolevatov, C. Pajares, V.V. Vechernin, Correlations between multiplicities and average transverse momentum in the percolating color strings approach, Eur. Phys. J. C 32 (2004) 535 [hep-ph/0307056].

[16] V.V. Vechernin, Long-range rapidity correlations in the model with independent emitters, in proceedings of the XX International Baldin Seminar on High Energy Physics Problems, vol.2, JINR, Dubna (2011) 10 [1012.0214 [hep-ph] ].

[17] V.V. Vechernin, Correlations between multiplicities in rapidity and azimuthally separated windows, 1210.7588 [hep-ph].

[18] V.V. Vechernin and H.S. Nguyen, Fluctuations of the number of participants and binary collisions in AA interactions at fixed centrality in the Glauber approach, Phys. Rev. C 84 (2011) 054909 [1102.2582 [hep-ph]].

[19] S. Jeon and V. Koch, Event by event fluctuations, in Quark-Gluon Plasma 3, World Scientific, Singapore (2004) 430 [hep-ph/ 0304012 ].

[20] C. Alt et al. (NA49 Collaboration), Centrality and system size dependence of multiplicity fluctuations in nuclear collisions at 158A GeV, Phys. Rev. C 75 (2007) 064904 [nucl-ex/ 0612010 ].

[21] A. Adare et al. (PHENIX Collaboration), Charged hadron multiplicity fluctuations in Au+Au and $\mathrm{Cu}+\mathrm{Cu}$ collisions from $\sqrt{s_{N N}}=22.5$ to $200 \mathrm{GeV}$, Phys. Rev. C 78 (2008) $044902[0805.1521$ [nucl-ex]].

[22] X. Artru, G. Mennessier, String model and multiproduction, Nucl. Phys. B 70 (1974) 93.

[23] X. Artru, Classical string phenomenology. How strings work, Phys. Rep. 97 (1983) 147.

[24] V.V. Vechernin, Space-time picture of the string fragmentation and the fusion of colour strings, in proceedings of the XIX International Baldin Seminar on High Energy Physics Problems, vol.1, JINR, Dubna (2008) $276[0812.0604$ [hep-ph] ]. 